\title{
Demokratie und Differenzierung bei Montesquieu
}

Wie modern Montesquieus politisches Denken zu seiner Zeit war und wie aktuell es bis heute geblieben ist, ist aus Anlass des zweihundertfünfzigjährigen Todestages des Barons ebenso häufig wie zurecht betont worden, ohne dass dies eine neue Erkenntnis gewesen wäre. Zuvorderst gilt dies für Montesquieus nachhaltige, konstruktive Wirkung auf den liberalen Konstitutionalismus. ${ }^{1}$ Dass hierzu Missverständnisse beziehungsweise Vereinnahmungen beigetragen haben, insbesondere was die Lehre von der Gewaltenverteilung angeht, ist bekannt. Für die politische Theorie und Ideengeschichte, die nicht nur nach argumentationslogischer und rezeptionsgeschichtlicher Korrektheit fragt, sind indessen gerade auch die Irrtümer, tendenziösen Interpretationen und Instrumentalisierungen Montesquieus bedeutsam, zeigen sie doch die Dynamik eines demokratischen Konstitutionalismus, der vielfältige Strömungen zu integrieren verstand. Die Besonderheit dieser Anschlussfähigkeit von Montesquieus, aus aristokratischem Geiste geborenem, liberalem Staatsdenken für den demokratischen Konstitutionalismus, und umgekehrt die Integrationsfähigkeit dieses Konstitutionalismus gegenüber Montesquieus liberalkonservativer Grundhaltung ${ }^{2}$ werden deutlich, wenn man sie mit der Rezeptionsgeschichte Jean-Jacques Rousseaus kontrastiert, die sich aus der Sackgasse des jakobinischen Terrors nie vollends zu befreien vermochte, wie unberechtigt der Totalitarismusvorwurf gegen die Idee der volonté générale auch sein mag. ${ }^{3}$ So wird man,

1 Neben der amerikanischen ist hier vor allem an die französische Verfassung von $1791 \mathrm{zu}$ denken, vgl. Elie Carcassonne, Montesquieu et le Problème de la Constitution Française au XVIII Siècle, Genève 1978; Anne M. Cohler, Montesquieu's Comparative Politics and the Spirit of American Constitutionalism, Lawrence, Kansas 1988 und Judith N. Shklar, Montesquieu, Oxford, New York 1987, S. 111ff., die Montesquieu als »the father of constitutions « preist.

2 Vgl. Cohler, Montesquieu's Comparative Politics and the Spirit of American Constitutionalism, aaO., (FN 1), S. 8; Stephen Holmes, »Differenzierung und Arbeitsteilung im Denken des Liberalismus« in: Niklas Luhmann (Hg.), Soziale Differenzierung. Zur Geschichte einer Idee, Opladen 1985, S. 9-41, 32; Emanuel Richter, Die Expansion der Herrschaft, Opladen 1994, S. 49. "Hat eine Verfassung eine seit langem bestehende Form, und haben die Dinge sich in einen bestimmten Zustand eingespielt, so ist es fast immer klug, sie zu belassen, weil die oftmals verwickelten und verdeckten Ursachen, die einen Staat erhalten haben, auch bewirken, daß er noch in Zukunft fortdauern wird. Ändert man jedoch das ganze System, so kann man nur den Unzulänglichkeiten abhelfen, die sich in der Theorie zeigen, und läßt andere bestehen, die allein die Praxis entdecken kann.« (Montesquieu, Größe und Niedergang Roms. Mit den Randbemerkungen Friedrichs des Großen. Übers. u. hg. v. Lothar Schuckert, Frankfurt a.M. 1980, S.115f.).

3 Jacob L. Talmon, Die Ursprünge der totalitären Demokratie, Köln 1961; Iring Fetscher: Rousseaus politische Philosophie. Zur Geschichte des demokratischen Freibeitsbegriffs, Frankfurt a.M. 1990, 258ff. 
cum grano salis, sagen können, dass Platon, Aristoteles, Machiavelli, Hobbes und Rousseau zwar häufiger und leidenschaftlicher diskutiert werden; doch niemand unter den Klassikern des politischen Denkens hatte so viel Einfluss auf den liberaldemokratischen Konstitutionalismus wie Montesquieu, der, ungeachtet seiner alteuropäischen Provenienz und Präferenzen, nahezu vorbehaltlos die strengen Kriterien posttotalitärer Ideologiekritik erfüllt.

Wie nachfolgend gezeigt wird, liegt ein wesentlicher Grund für diesen historischen Erfolg Montesquieus in seiner Ergänzung einer normativen durch eine funktionale Perspektive (1.). Dies bedeutet, Montesquieus liberales Staatsdenken auf seine politiksoziologische Perspektive zurückzubeziehen, die, neben seiner Regimelehre, ein weiteres, seit längerem bekanntes, aber immer wieder aufs Neue gepriesenes Modernitäts- und Aktualitätskriterium bildet. Bereits Durkheim hatte Montesquieu zusammen mit Rousseau als »Vorläufer der Soziologie « vereinnahmt, was in dieser Hinsicht zutrifft, aber eine Nähe zwischen dem Aristokraten aus Bordeaux und dem Autodidakten aus Genf insinuiert, die nur um den Preis der Ausblendung aller vorstehend angesprochenen, politischen und rezeptionsgeschichtlichen Gegensätze möglich und daher problematisch ist.

Anstelle dessen wird hier argumentiert, dass Montesquieus Politische Soziologie Elemente einer Theorie sozialer Differenzierung enthält (2.). Diese bestimmt auch seine Gewaltenverteilungslehre, insofern sie Rechtsfunktionen spezifiziert, die Montesquieu in den ersten Kapiteln des XXVI. Buches Vom Geist der Gesetze ausdrücklich von solchen der Religion und der Politik unterscheidet. Vervollständigt wird diese Differenzierungstheorie nicht nur von Montesquieus Überzeugung, dass der Ökonomie eine separate, eigengesetzliche Funktion im Gesellschaftsgefüge zukommt, sondern auch von seinem spezifisch liberalen Öffentlichkeitskonzept. Sein hierin deutliches Verständnis von sozialer Integration ermöglicht Montesquieu eine Differenzierung der Demokratisierungsfrage (3.). Dieser Ansatz einer Moderation von Modernisierungsprozessen spiegelt sich in seiner Regimetypologie wider und begründet nicht nur seine Divergenz gegenüber Aristoteles, sondern auch ein neues, arbeitsteiliges Verhältnis zwischen Regierungslehre und politischer Theorie, in dem ein aktuelles Verständnis von Demokratie und Staatlichkeit aufscheint.

\section{Zwischen Politischer Theorie und Politischer Soziologie}

Es war ein kleiner Schritt für Montesquieu, aber ein großer Schritt für die politische Ideengeschichte, innerhalb der normativen politischen Theorie eine politiksoziologische Perspektive zu entwickeln. Vielleicht ist dies bis dato im Schatten der Montesquieu-Rezeption verblieben, weil damit zwei nach heutigen Vorstellungen schwer vereinbare Ansätze kombiniert wurden. Nur jene Verknüpfung aber ermöglichte es der republikanischen Staatsauffassung, aus ihrer babylonischen Gefangenschaft in einer rein normativen politischen Theorie herauszutreten und sich in Richtung des demokratischen Konstitutionalismus gleichzeitig zu konkretisieren wie zu

4 Emile Durkheim, Montesquien et Rousseau. Précurseurs de la sociologie, Paris 1966. 
liberalisieren. Bei Montesquieu ist dies verbunden mit der Einnahme einer sich an der Schnittstelle zwischen Methodik und Normativität entscheidenden Äquidistanz gegenüber den beiden großen Antipoden Aristoteles und Hobbes.

So unterscheidet sich Montesquieus Ansatz von Aristoteles' Regimetypologie, indem das Kriterium quantitativer Machtverteilung differenziert und das für Aristoteles zentrale Kriterium des qualitativen Herrschaftszweckes modifiziert wird. An die Stelle des Gemeinwohls tritt die rule of law, die Herrschaft nach Recht und Gesetz. »Montesquieu war an shöchsten Zwecken< uninteressiert. [...] Die legitimierte Autorität hat kein Ideal, das sie durchsetzen kann, oder ein Fundament, auf dem sie aufruht, sondern lediglich ein Problem, das sie lösen kann. $\aleph^{5}$ So folgt Montesquieu zwar noch insofern dem aristotelischen Ansatz, als er die Überlebensfähigkeit freiheitlicher Ordnungen mit ihren sozio-moralischen Qualitäten korreliert, doch kulminieren diese Überlegungen in der Sorge um die Funktionsfähigkeit groBer Gesellschaften. Die normative politische Theorie führt mithin zur »sozialen Morphologie« und die Prinzipientheorie zur Organisationssoziologie. ${ }^{6}$

Ein berühmtes Beispiel hierfür ist das Gleichnis von den Troglodyten aus Montesquieus Persischen Briefen ${ }^{7}$ : Die Troglodyten, ein animalisches, wildes und bösartiges Volk, haben, jeglicher Herrschaft und Gesellschaftsordnung überdrüssig, ihre Herrscher getötet und daraufhin beschlossen, Individualinteresse und Eigennutzenmaximierung zum alleinigen Handlungsprinzip zu machen. Folgerichtig weigerten sie sich nach einer Epidemie, den sie heilenden Arzt zu entlohnen, so dass dieser nach dem erneuten Ausbruch der Krankheit die Behandlung verweigerte, woraufhin alle Troglodyten bis auf zwei Familien ausstarben. Diese entsprachen nicht dem Mehrheitsgeschmack:

»Sie besaßen Menschlichkeit, kannten Gerechtigkeit, liebten die Tugend. [...] Ihre ganze Aufmerksamkeit galt der Erziehung ihrer Kinder zur Tugend. [...] Sie machten ihnen vor allem verständlich, daß das Interesse der einzelnen im Interesse der Gemeinschaft liegt, daß es den eigenen Untergang bedeutet, wenn man sich von ihr trennen möchte, daß die Tugend uns keine Mühe machen darf [...] und daß Gerechtigkeit gegen andere eine Wohltat für uns selbst bedeutet. «

So gelingt dieser durch ihre politische Tugendhaftigkeit ausgezeichneten, zweiten Generation der Troglodyten die Abwehr äußerer Feinde und die Sicherung des Gemeinwesens, das solange als Republik zu existieren vermag, bis es ein zu starkes Bevölkerungswachstum aufweist und zu dessen eigenem Kummer ein König gewählt wird.

Diese parabolische Geschichte aus dem Frühwerk enthält bereits wesentliche Grundzüge der Überlegungen, die Montesquieu später unter vielfacher Bezugnah-

5 Holmes, Differenzierung und Arbeitsteilung im Denken des Liberalismus, aaO. (FN 2), S. 36 (Anm. 18).

6 Raymond Aron, Hauptströmungen des soziologischen Denkens. Band I: Montesquieu, Auguste Comte, Karl Marx, Alexis de Tocqueville, Köln 1965, S. 30.

7 Charles-Louis de Secondat, Baron de la Brède et de Montesquieu, Persische Briefe. Übers. u. hg. v. Peter Schunck, Stuttgart 1991, 11.-14. Brief.

8 Montesquieu, Persische Briefe, aaO. (FN 7), S. 32. 
me auf die griechischen und römischen Erfahrungen zu einem skeptischen Urteil hinsichtlich der Realisierbarkeit einer republikanischen Demokratie weiterentwickelt. Als Grund hierfür gibt er an, dass monarchische oder despotische Regierungen einer sozio-moralischen »Triebkraft « entraten könnten, nicht indessen ein auf die Tugend $«$ der Regierten vital angewiesener $»$ Volksstaat $« .{ }^{9}$ Anders aber als Aristoteles, für den der unauflösliche Zusammenhang zwischen Politik und Moral die normative Richtungsanzeige der politischen Theorie bildet, anders aber auch als beispielsweise Machiavelli, der die sozio-moralische Ressource der virtù dem ewigen Auf und $\mathrm{Ab}$ der menschlichen Dinge überantwortet sieht und die normative Theorie damit geschichtsphilosophisch grundiert, kommt Montesquieu zu dem Ergebnis, dass die sozio-moralischen Qualitäten manifesten, quantifizierbaren, strukturellen Determinanten unterliegen. Republiken sind demnach, wie im Fall der Troglodyten, nur überlebensfähig, solange sie territorial überschaubar bleiben, weil allein dies die motivationale Voraussetzung von Gemeinsinn erfüllt, dass nämlich das Gemeinwohl überhaupt erkennbar, das heißt lebensweltlich konkretisierbar bleibt.

»Es gehört zum Wesen der Republik, daß sie nur ein kleines Gebiet umfaßt, sonst kann sie nicht bestehen. In einer großen Republik gibt es große Vermögen und infolgedessen wenig Sinn für Mäßigung. [...] In einer großen Republik wird das Gemeinwohl tausenderlei Rücksichten geopfert, es unterliegt Ausnahmen und hängt von Zufällen ab. In einer kleinen Republik dagegen wird das Gemeinwohl stärker empfunden, besser erkannt, dem einzelnen Bürger näher gerückt «. ${ }^{10}$

In dieser Lesart Montesquieus bilden die Persischen Briefe »an embryonic comparative political sociology of contemporary society «, ${ }^{11}$ an die sich werkgeschichtlich die Betrachtungen über die Ursachen von Größe und Niedergang der Römer als einer »methodological exercise in comparative sociology « anschließen, die wiederum der Vorbereitung des Geists der Gesetze diente, in dem »Montesquieu's gradual progression towards a sociological perspective $\ll^{12}$ zum Abschluss kam. Dies zeigt sich, wenn man mit Raymond Aron die Bücher I-XIII als allgemeine Politische Soziologie versteht, denen in den Büchern XIV-XIX eine materielle und physische Ursachentheorie und in den Büchern XX-XVI eine soziale Ursachentheorie folgt. Diese wiederum wird im IXX. Buch einem »Vereinheitlichungsprinzip des sozialen Ganzen « ${ }^{13}$ zugeführt, wobei Montesquieus bedächtiger, anti-revolutionärer Reformismus in der Maxime, man müsse nicht alles verändern wollen, ${ }^{14}$ ebenso deutlich wird wie sein Schritt zum Anti-Moralismus in dem Hinweis, er habe am Beispiel der klimatisch und geographisch bedingten, merkantilen Unredlichkeit der Chine-

9 Montesquieu, Vom Geist der Gesetze. Übers., eingel. u. hg. v. Ernst Forsthoff, 2 Bde., Tübingen ${ }^{2} 1992$, Bd. I, S. 34.

10 Montesquieu, Vom Geist der Gesetze, aaO. (FN 9), Bd. I, S. 172.

11 John Alan Baum, Montesquieu and Social Theory, Oxford 1979, S. 49.

12 Baum, Montesquieu and Social Theory, aaO. (FN 11), S. 74.

13 Aron, Hauptströmungen des soziologischen Denkens, aaO. (FN 6), S. 26.

14 Montesquieu, Vom Geist der Gesetze, aaO. (FN 9), Bd. I, S. 414. 
sen »zeigen wollen, daß nicht alle politischen Laster auch moralische, und dass nicht alle moralischen Laster auch politische $\operatorname{sind} \ll .{ }^{15}$

Gegenüber Montesquieus Abgrenzung von Aristoteles ist diejenige von Hobbes, wie eingangs betont, nicht minder deutlich, ${ }^{16}$ und zwar ebenfalls dank Montesquieus methodischer Modernität. ${ }^{17}$ Geradezu im Sinne von Foucaults fröhlicher Wissenschaft des Judo enthebt sie ihn nämlich einer offenen Zurückweisung des Hobbes'schen Szientismus einer politischen Theorie more geometrico, wie auch einer Akzeptanz von dessen kryptonormativem, antirepublikanischem Autoritarismus. Montesquieu eröffnet ein neues Feld: Mit seiner, bei aller konventionellen Klimatologie, avancierten Perspektive, dass die sozialstrukturellen Bedingungen über den Erfolg und also über den Sinn einer Regierungsform entscheiden, vermag er das Plädoyer für eine konstitutionelle Republik von der durch Hobbes angegriffenen normativen politischen Theorie zu trennen und seinerseits der more geometrico den szientistischen Glaubwürdigkeitskredit zu kürzen. Ratio, neque veritas, neque auctoritas facit legem, müsste Montesquieus Formel lauten. Denn »Montesquieu veut fonder son action politique sur une science sociale certaine et précise «. ${ }^{18}$

Diese Innovation ermöglicht Montesquieu aber nicht nur Äquidistanz gegenüber den zu seiner Zeit schon traditionellen politiktheoretischen Antipoden Aristoteles und Hobbes, sondern sie markiert auch die Diskrepanz gegenüber Jean-Jacques Rousseau. Denn entgegen Rousseaus radikaldemokratischem Republikanismus ermöglichte Montesquieus politiksoziologische Ergänzung normativer politischer Theorie die Weiterentwicklung der republikanischen Staatsauffassung zum liberalen Konstitutionalismus. Zwischen Mitte und Ende des 18. Jahrhunderts trennen sich mithin sowohl theorie- wie auch realgeschichtlich die Wege zwischen einem moderaten, reformistischen, liberalen Konstitutionalismus, wie er von Montesquieu vertreten und in den Vereinigten Staaten von Amerika praktiziert wurde, einerseits, und einem emphatisch-progressistischen, egalitären Radikalismus, wie er von Rousseau vertreten und in der Französischen Revolution pervertiert wurde.

Wie eingangs in Aussicht gestellt, soll Montesquieus Entwurf hier aber nicht bloß als Determinante seines liberalen Staatsdenkens herausgestellt werden, sondern als Frühform einer Differenzierungstheorie interpretiert werden, die nicht nur Montesquieus demokratietheoretische Vorstellungen erklären hilft, sondern auch unsere heutigen aufzuklären vermag.

15 Montesquieu, Vom Geist der Gesetze, aaO. (FN 9), Bd. I, S. 418.

16 Vgl. Simone Goyard-Fabre, Montesquieu: la Nature, les Lois, la Liberté, Paris 1993, S. 85ff.; Baum, Montesquieu and Social Theory, aaO. (FN 11), S. 45.

17 Louis Althusser, Montesquieu. La politique et l'histoire, Paris 1959, S. 11ff. spricht von "une révolution dans la méthode«, und Mark Hulliung, Montesquieu and the Old Regime, Berkeley u.a. 1976, S. 25ff. sieht »Political Sociology as the Indictment of Absolutism« und attestiert Montesquieu gar »a >Copernican revolution< in political thought, comparable to Kant's >Copernican revolution in philosophy (S. 221).

18 Charles-Jacques Beyer, »Le problème du déterminisme social dans l'Esprit des lois « in: The Romanic Review, Vol. XXXIX, Nr. 1 (1948), S.102-106, 104. 
Die Voraussetzung hierfür ist Montesquieus Abwendung von jedwedem essentialistischen oder gar organizistischen Gesellschaftsbegriff. So heißt es in dem völkerrechtlichen Kontext des dritten Kapitels vom X. Buch des Geists der Gesetze, die Gesellschaft sei »die Vereinigung von Menschen und nicht dasselbe wie Menschen als solche « ${ }^{19}$ Was Montesquieu interessiert, sind die sozialstrukturellen Mechanismen dieser Vereinigung: »By viewing society as a set of interrelated elements, Montesquieu was putting forward a holistic interpretation of social structure akin to that which today we know as functionalism $\ll{ }^{20}$ Folgerichtig fokussiert dieser Ansatz die »functional interdependence of institutions «, was für die zentrale Bedeutung der Gewaltenverteilungslehre spricht. ${ }^{21}$ In diesem Vorrang politischer Machtbeschränkung (constraint) durch soziale Institutionen gegenüber moralischen Intentionen erweist sich der moderne Liberalismus Montesquieus, ${ }^{22}$ und zwar in dem gerade erörterten, engen Zusammenhang mit seiner politiksoziologisch innovativen Perspektive als Dreh- und Angelpunkt. Diese nämlich, und nicht etwa Montesquieus Konservatismus oder methodische Rückständigkeit, verhindert wiederum, dass seine Theorie ein weiteres Kennzeichen des modernen Liberalismus erfüllt, und zwar die Unterscheidung zwischen »Staat« und »Gesellschaft«. Montesquieu interessiert die jenseits dieser Unterscheidung liegende, normativ aber nicht minder liberal motivierte Frage nach den Bedingungen für die Herstellung einer Einheit des Differenten, und auf nichts anderes richtet sich der Differenzierungsbegriff. $^{23}$

\section{Montesquieus Differenzierung der Demokratisierung}

Bekanntlich verdankt Montesquieu seinen Ruhm einer Differenzierungsidee: der »Gewaltenteilung auf Grund rationaler Differenzierung der Staatsfunktionen «.24 Deren eingangs bereits in ihrer Eigenbedeutung angesprochene, bewegte Rezeptionsgeschichte bedarf hier keiner erneuten Rekapitulation. ${ }^{25}$ Für den vorliegenden Zusammenhang entscheidend ist der erstmalig von Charles Eisenmann betonte Umstand, dass das Konzept der Gewaltenverteilung auf die Garantie der Herrschaft des Gesetzes (»le règne de la loi«) gerichtet ist. Montesquieu geht es um die rule of law: »Le principe de la séparation des pouvoirs répond simplement au soici d'assurer la légalité de l'exécution « ${ }^{26}$ Seine Gewaltenverteilungslehre differenziert also nicht primär "Staatsorgane, sondern Rechtsfunktionen, d.h. juristisch gekennzeich-

19 Montesquieu, Vom Geist der Gesetze. Eingel., ausgew. u. übers. v. Kurt Weigand, Stuttgart 1965, S. 203. Forsthoffs Übersetzung des originalen »La société est l'union des hommes, et non pas les hommes « (Montesquieu, Euvres Complètes. Texte présenté et annoté par Roger Caillois, 2 Bde., Paris 1951, Bd. II, S. 379) ist hier weniger treffend: »Die Gemeinschaft ist ein Verband von Menschen und nicht die einzelnen Menschen« (Montesquieu, Vom Geist der Gesetze, aaO. (FN 9), Bd. I, S. 193).

20 Baum, Montesquieu and Social Theory, aaO. (FN 11), S. 91.

21 Baum, Montesquieu and Social Theory, aaO. (FN 11), S. 84.

22 Stephen Holmes, Passions and Constraint. On the Theory of Liberal Democracy, Chicago, London 1995, S. 13, 101.

23 Niklas Luhmann, Die Gesellschaft der Gesellschaft, Frankfurt a.M. 1997, S. 595.

24 Max Imboden, Montesquieu und die Lebre der Gewaltenteilung, Berlin 1959, S. 11. 
nete Formen der Staatstätigkeit «. ${ }^{27}$ Dabei wird jeder der drei Gewalten ein anderes Prinzip zugedacht: In der Legislative sollen demokratische Formen dominieren, in der Exekutive monokratische und in der Judikative aristokratische. ${ }^{28}$ Hierin zeigt sich Montesquieus konservativ-reformistisches Interesse an einer sozialen Verteilung der Gewalten zwischen Monarch, Adel und Repräsentanten des Bürgertums. Diese bildet für ihn eine unerlässliche Voraussetzung der institutionellen Gewaltenverteilung, der allein er keine hinreichende Freiheitsgarantie zutraut. ${ }^{29}$

Die soziale Verteilung der Gewalten verspricht sich Montesquieu aber eben nicht bloß vom Verhältnis zwischen den drei Ständen, sondern von einem gesamtgesellschaftlichen Differenzierungsschub funktionaler Art. Demnach kommt den für die Gewaltenverteilung ausschlaggebenden Rechtsfunktionen eine Eigenlogik zu, die im XXVI. Buch des Geists der Gesetze von derjenigen der Religion und derjenigen der Politik gleichermaßen unterschieden wird. Und schon zuvor, im XX.-XXII. Buch, beschreibt Montesquieu die Eigenlogik der Wirtschaft, gegenüber deren Profitorientierung er zwar »eine aristokratische Geringschätzung « kultivierte, deren Autonomie er aber gleichwohl als Schutzwall insbesondere gegenüber religiös motivierten Kriegen hochschätzte ${ }^{30}$ und zum Anlass eines Plädoyers für internationalen Freihandel nahm. Dabei geht diese liberale Überzeugung von der ökonomischen Eigengesetzlichkeit und der entsprechenden Dysfunktionalität politischer Einmischung $^{31}$ zurück auf Montesquieus liberales Staatsdenken, denn er sieht einen Zusammenhang zwischen der Zeichen- und Repräsentationsfunktion des Geldes und seinem Ideal einer gemäßigten Regierung. Nur unter dieser, so heißt es im 2. Kapitel des XXII. Buches Vom Geist der Gesetze, komme es dazu, dass »das Geld alle Sachen vertritt und alle Sachen das Geld vertreten « und also der Wohlstand des Staates

25 Vgl. Imboden, Montesquieu und die Lehre der Gewaltenteilung, aaO. (FN 24); Alois Riklin, "Montesquieus freiheitliches Staatsmodell. Die Identität von Machtteilung und Mischverfassung « in: Politische Vierteljabresschrift 30, 1989, S. 420-442; ders., »Die gewaltenteilige Mischverfassung Montesquieus im ideengeschichtlichen Zusammenhang in: Paul-Ludwig Weinacht (Hg.), Montesquieu. 250 Jabre »Geist der Gesetze". Beiträge aus Politischer Wissenschaft, Jurisprudenz und Romanistik, Baden-Baden 1999, S. 15-29; Detlef Merten, »Montesquieus Gewaltenteilungslehre und deutsche Verfassungsstaatlichkeit « in: Paul-Ludwig Weinacht (Hg.), Montesquieu. 250 Jabre »Geist der Gesetze». Beiträge aus Politischer Wissenschaft, Jurisprudenz und Romanistik, Baden-Baden 1999, S. 31-62.

26 Charles Eisenmann, »L’ >Esprit des lois« et la séparation des pouvoirs « in: Mélanges R. Carré de Malberg, Paris 1933, S. 163-192, 190. Vgl. auch Althusser, Montesquieu. La politique et l'histoire, aaO. (FN 17), S. 98ff.

27 Imboden, Montesquieu und die Lehre der Gewaltenteilung, aaO. (FN 24), S. 11.

28 Imboden, Montesquieu und die Lehre der Gewaltenteilung, aaO. (FN 24), S. 16.

29 Vgl. Iring Fetscher, »Politisches Denken im Frankreich des 18. Jahrhunderts vor der Revolution « in: ders. / Herfried Münkler (Hg.), Pipers Handbuch der politischen Ideen. Band 3: Neuzeit: Von den Konfessionskriegen bis zur Aufklärung, München 1985, S. 423-528, 452.

30 Holmes, Differenzierung und Arbeitsteilung im Denken des Liberalismus, aaO. (FN 2), S. 16.

31 Holmes, Differenzierung und Arbeitsteilung im Denken des Liberalismus, aaO. (FN 2), S. 16. 
innerhalb eines transparenten, das heißt von einem Publikum beobachtbaren Wirtschaftskreislaufs gesichert wird. ${ }^{32}$ Damit ist zugleich das Modell liberaler Öffentlichkeit entworfen, innerhalb dessen der öffentlichen Meinung eine Ausgleichsfunktion zukommt, ohne moralisch überdeterminiert zu sein. So heißt es im 27. Kapitel des XIX. Buches Vom Geist der Gesetze, »in einem freien Volk « sei »es sehr häufig ganz gleichgültig, ob die einzelnen Gutes oder Schlechtes reden; es genügt, daß sie reden: hieraus entspringt die Freiheit, die vor den Folgen solcher Reden selbst schützt. «33 Die öffentliche Meinung ist also für Montesquieu ein sozialer Machtfaktor, dessen Funktionalität nicht bloß zuverlässiger und berechenbarer ist als jedes normative Postulat es vermöchte. Darüber hinaus schreibt er ihr einen Einfluss auf Politik, Wirtschaft, Recht und Religion zu, der mit einer stratifizierten Sozialordnung unvereinbar ist. Auf diese Weise verdrängt das liberale Modell funktionaler Sozialdifferenzierung mit seiner Kontrastierung zwischen öffentlicher und privater Sphäre »den viel verhaßteren Gegensatz zwischen den sozialen Schichten. «34 Insofern also das »Gleichgewicht der sozialen Kräfte « für Montesquieu die $»$ Vorbedingung der politischen Freiheit ${ }^{35}$ darstellt und dieses über einen Klassenkompromiss hinausgehende Gleichgewicht auf der Ausdifferenzierung gesellschaftlicher Funktionen basieren soll, lässt sich feststellen, dass Montesquieu die ihm aus England vertraute Idee der institutionalisierten checks and balances in Richtung eines für sämtliche Institutionen grundlegenden, sozialen Ordnungskonzeptes weitergedacht hat. Mit anderen Worten: Montesquieus Gewaltenverteilungslehre beruht auf der Einsicht, dass die Stratifikation als soziale Primärdifferenzierung historisch überholt und durch die Orientierung an gesellschaftlichen Funktionen zu ersetzen ist. Dies verweist bereits auf die spätere Einsicht, dass nach dem Veralten sozialer Stratifizierung ein wechselseitiges Bedingungsverhältnis zwischen funktionaler Gesellschaftsdifferenzierung und konstitutioneller Demokratie entstanden ist. So ist Montesquieu überzeugt, dass freiheitliche Regime des Aufbaus und der Bewahrung sozialer Komplexität bedürfen, während die simple Struktur von Despotien auf ebensolchen Sozialstrukturen ruht. ${ }^{36}$ Man kann demnach Montesquieu die Vordenkerschaft für die Überzeugung der heutigen Gesellschaftstheorie attestieren, dass Komplexität und Demokratie positiv korrelieren. ${ }^{37}$

Diese Auffassung resultiert aus Montesquieus »Versuch einer vernünftigen Einbindung des Politischen in ein Gesamtmodell des sozialen Systems « ${ }^{38}$ dem eine nicht-deterministische Vorstellung von sozialer Integration zugrunde liegt. ${ }^{39}$ So

32 Montesquieu, Vom Geist der Gesetze, aaO. (FN 9), Bd. II, S. 84.

33 Montesquieu, Vom Geist der Gesetze, aaO. (FN 9), Bd. I, S. $442 f$.

34 Holmes, Differenzierung und Arbeitsteilung im Denken des Liberalismus, aaO. (FN 2), S. 19.

35 Aron, Hauptströmungen des soziologischen Denkens, aaO. (FN 6), S. 36.

36 Nannerl O. Keohane, Philosophy and the State in France. The Renaissance to the Enlightenment, Princeton, New Jersey 1980, S. 398.

37 Kai-Uwe Hellmann / Karsten Fischer, »Einleitung: Niklas Luhmanns politische Theorie in der politikwissenschaftlichen Diskussion « in: Dies. / Harald Bluhm (Hg.), Das System der Politik. Niklas Lubmanns politische Theorie, Wiesbaden 2003, S. 9-16, 13. 
heißt es in den Betrachtungen über die Ursachen von Größe und Niedergang der Römer:

»Was man bei einem politischen Gebilde als Einheit bezeichnet, ist eine sehr unbestimmte Sache. Die wahre Einheit ist harmonische Einheit, die bewirkt, daß alle Teile, so entgegengesetzt sie auch immer erscheinen mögen, zum allgemeinen Wohle der Gesellschaft beitragen, so wie alle Dissonanzen in der Musik sich zum totalen Akkord vereinigen. Es kann in einem Staate, in dem man nur Unruhe wahrzunehmen glaubt, Einheit geben, d.h. eine Harmonie, aus der das Glück entspringt, das allein der wahre Friede ist. Es ist damit wie mit den Teilen dieses Weltganzen, die durch actio und reactio in Ewigkeit verbunden sind. «40

Montesquieu denkt also, auch hierin ganz modern, Sozialintegration als Einheit des Differenten, die sich über fortschreitende Differenzierung, und nicht etwa infolge deren Hemmung, einstellt. ${ }^{41}$

Der bis hierhin rekonstruierte Zusammenhang zwischen Differenzierung und Demokratisierung bei Montesquieu ist von maßgeblicher Bedeutung für seine Regimelehre und Demokratietheorie, hinsichtlich derer sich die Distanzierung von Aristoteles erneut und typologisch verdichtet zeigt.

Bekanntlich hatte Aristoteles in seiner Regimetypologie eine quantitative und eine qualitative Ebene relationiert: ${ }^{42}$

38 Effi Böhlke, »Esprit de nation«. Montesquieus politische Philosophie, Berlin/BadenBaden 1999, S. 211.

39 Vgl. Werner Stark, Montesquieu. Pioneer of the Sociology of Knowledge, London 1960, S. 72. Anders Goyard-Fabre, Montesquieu: la Nature, les Lois, la Liberté, aaO. (FN 16), S. 289: »La répresentation des citoyens étant en même temps la répresentation des partis et de leurs volontés spécifiques, la dynamique politique ne saurait se réduire au méchanisme des forces et des contre-forces de la nation: elle en exprime la vie profonde et l'esprit général: l'Etat représente la société. «

40 Montesquieu, Größe und Niedergang Roms, aaO. (FN 2), S. 59.

41 Vgl. Luhmann, Die Gesellschaft der Gesellschaft, aaO. (FN 23), S. 595.

42 Aristoteles, Politik. Übers. u. hg. v. Olof Gigon, München 1986, S. $113 \mathrm{ff}$. 


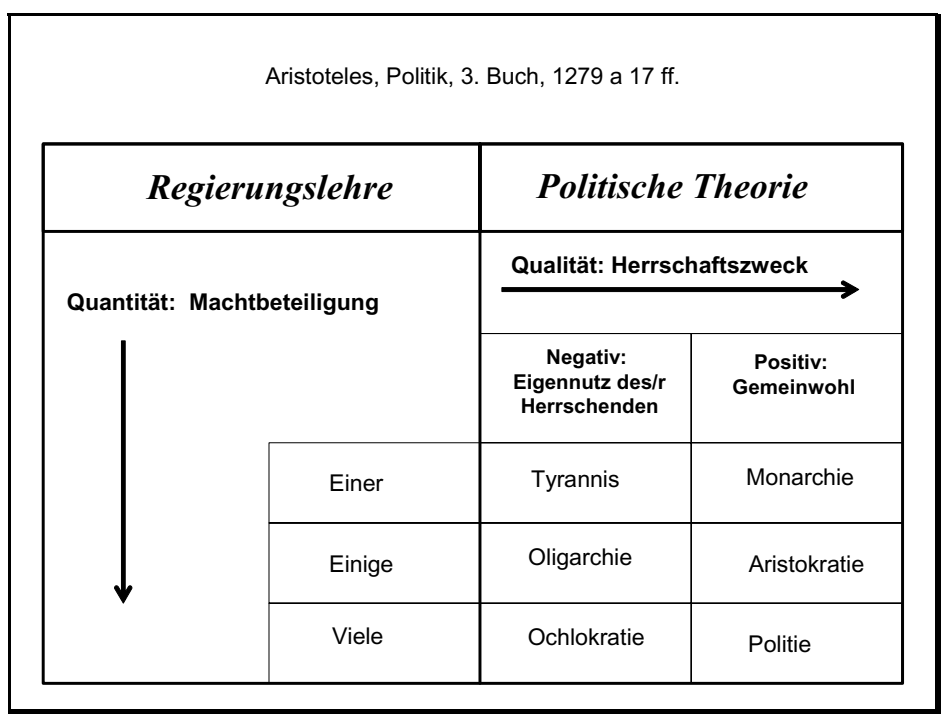

Demnach ist die quantitative Frage der Machtverteilung, das heißt die Frage der Machtbeteiligung, wie sie sich, in heutigen Termini, der Regierungslehre stellt, ${ }^{43}$ verwiesen auf die nach heutigen Begriffen der Politischen Theorie zugehörige, qualitative Frage nach dem Herrschaftszweck, hinsichtlich derer sich die Alternative stellt, ob die Herrschaft dem Gemeinwohl oder dem Eigennutz des beziehungsweise der Herrschenden dienen soll. Erst hierdurch vermag Aristoteles die Regimetypen zu bestimmen, was ihre normative Beurteilung gleichzeitig mitbestimmt.

Montesquieu hingegen differenziert dieses Schema, indem er die Perspektive einer Politischen Soziologie zur Geltung bringt und mit derjenigen der Politischen Theorie so kombiniert, dass ein erheblicher Komplexitätsgewinn entsteht. So wird zunächst der anti-liberalen Lesart Thomas Hobbes' en passant der Boden entzogen. Hobbes hatte die aristotelische Typologie auf die quantitative Ebene reduziert und die qualitative Frage, ob etwa die Herrschaft eines Einzelnen Monarchie oder Tyrannis sei, als bloßes Symptom für die Bedeutung der Semantik im politischen Machtkampf ausgegeben: ${ }^{44}$

43 Cohler, Montesquieu's Comparative Politics and the Spirit of American Constitutionalism, aaO. (FN 1).

44 Thomas Hobbes, Leviathan oder Stoff, Form und Gewalt eines kirchlichen und bürgerlichen Staates. Hg. u. eingel. v. Iring Fetscher, übers. v. Walter Euchner, Frankfurt a.M. 1991, S. 145. 


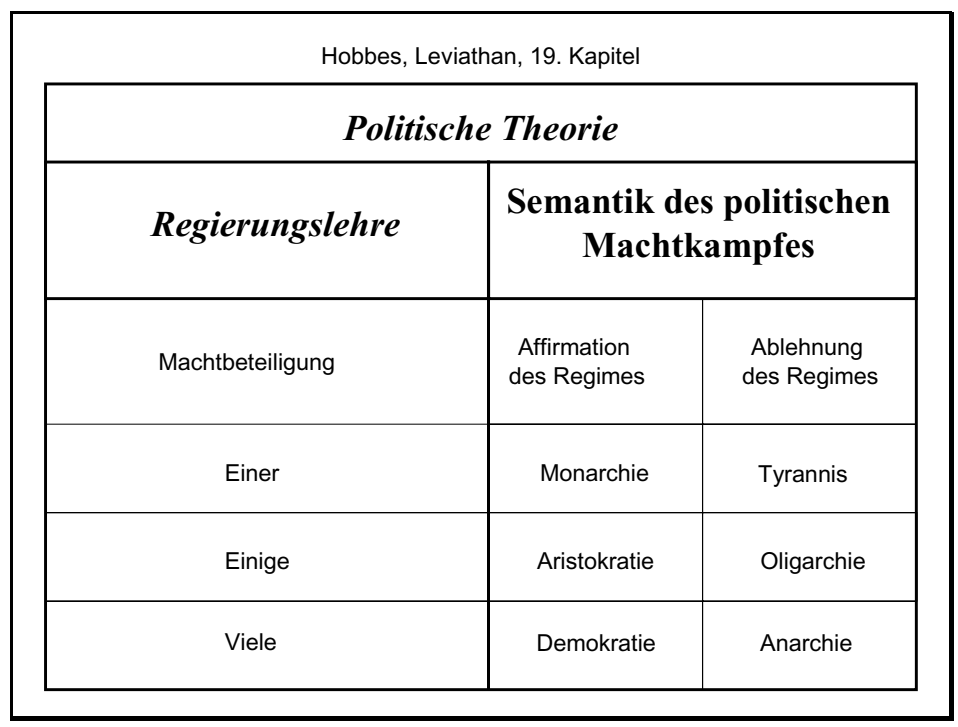

Montesquieu jedoch verzichtet in seiner Regimetypologie auf das normative Kriterium des Herrschaftszweckes und entgeht damit der Hobbes'schen Semantologie. Vielmehr stellt Montesquieu seit der Geschichte von den Troglodyten darauf ab, dass die Regimetypen auch und gerade hinsichtlich der für ihren Bestand unverzichtbaren sozio-moralischen Qualitäten mit sozialstrukturellen Aspekten korrelieren, ${ }^{45}$ deren grundlegendster das Komplexitätsniveau der funktionalen Primärdifferenzierung ist. Dabei dient die Verteilung der Gewalten einer Austarierung der sozialen Kräfte. Einer demokratisch dominierten Legislative stehen eine monokratische Exekutive und eine aristokratisch geprägte Judikative gegenüber. ${ }^{46}$ Auf diese Weise erhofft sich Montesquieu eine Differenzierung der Demokratisierung mit Nutzen in beiderlei Hinsicht: Einerseits steigen durch die Aufrechterhaltung traditioneller Regierungselemente die Chancen einer friedfertigen, reformerischen Demokratisierung; andererseits ist es ein Hauptanliegen von Montesquieu, dass schlechthin keine Gewalt, nicht einmal die des Volkes über sich selbst, unbegrenzt sein darf. ${ }^{47}$ Wenn aber die drei klassischen Staatsformen, Monarchie, Aristokratie und Demokratie, solchermaßen »zu Attributen der innerstaatlichen Gewalten «, Exekutive, Judikative und Legislative, weiterentwickelt sind, taugen ihre »Formprinzipien « nicht mehr zur generalisierenden Kennzeichnung des Regimetypus. ${ }^{48}$ Folglich nimmt Montesquieu im II., III. und VIII. Buch des Geists der Gesetze eine Neukonzeption der Regimetypologie vor:

45 Vgl. Aron, Hauptströmungen des soziologischen Denkens, aaO. (FN 6), S. 30.

46 Imboden, Montesquieu und die Lehre der Gewaltenteilung, aaO. (FN 24), S. 16.

47 Vgl. Aron, Hauptströmungen des soziologischen Denkens, aaO. (FN 6), S. 38.

48 Imboden, Montesquieu und die Lehre der Gewaltenteilung, aaO. (FN 24), S. 18. 


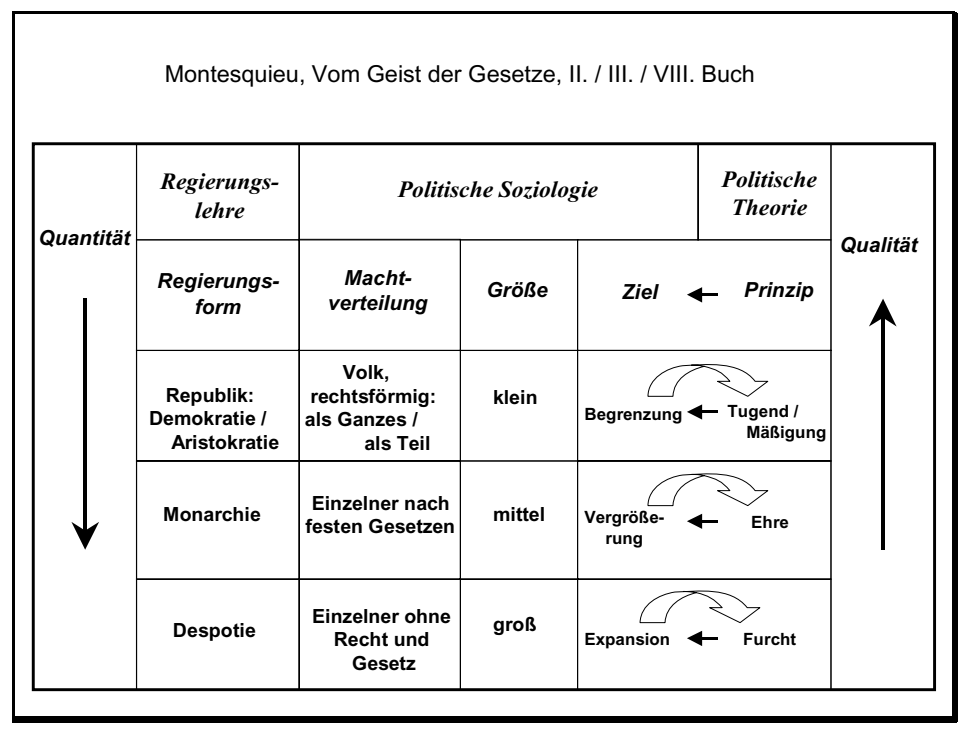

Dabei übernimmt er die Hobbes'sche Reduktion der aristotelischen Typologie von sechs auf drei Regierungsformen, wobei er diese jedoch anders kontrastiert. Und zwar vereinheitlicht er Demokratie und Aristokratie als zwei nur in puncto Machtbeteiligung unterschiedliche Erscheinungsformen der Republik. Dieser gegenüber steht nicht nur, wie gewohnt, die Monarchie, sondern nun auch die Despotie, die bei Aristoteles nur auf der negativen Seite der Unterscheidung aufgetaucht und bei Hobbes gänzlich der ihrerseits rhetorisch-polemischen, ${ }^{49}$ semantologischen Perspektive zum Opfer gefallen war.

Wie Raymond Aron bemerkt hat, liegt der tiefere Grund dieses Anti-Aristotelismus in Montesquieus Politischer Soziologie, mit der die »Unterscheidung der Regierungsformen « stets auf die »Unterscheidung der sozialen Organisationen und Strukturen« zurückbezogen wird. Während nämlich für Aristoteles die drei klassischen Regierungsformen »der politische Überbau einer bestimmten Gesellschaftsform waren «, deren Beziehung zur sozialen Basis kaum reflektiert wurde, reformuliert Montesquieu die gesamte Problemstellung und verbindet Regimetypen und Gesellschaftsanalyse, indem er auf die soziale Morphologie abstellt und im Rahmen dessen seine bereits erläuterte Überzeugung anbringen kann, dass Republiken nur ein kleines Gebiet umfassen dürfen..$^{50}$

Gleichzeitig wird sowohl das quantitative als auch das qualitative Kriterium der aristotelischen Regimetypologie kontextualisiert, indem Montesquieu auch hier die Perspektiven der Regierungslehre und der Politischen Theorie um die Sicht der Po-

49 Vgl. Matthias Bohlender, Die Rhetorik des Politischen. Zur Kritik der politischen Theorie, Berlin 1995, S. $79 \mathrm{ff}$.

50 Aron, Hauptströmungen des soziologischen Denkens, aaO. (FN 6), S. 29. 
litischen Soziologie ergänzt. So wird die Frage der quantitativen Machtverteilung um das letztlich qualitative, gegenüber dem Postulat der Gemeinwohlorientierung aber normativ anspruchslosere Kriterium der rule of law ergänzt, das den entscheidenden Unterschied zwischen der gesetzesförmigen Monarchie und der gesetzlosen Despotie ausmacht und die conditio sine qua non einer Republik darstellt.

Diese Positionierung ersetzt aber bei Montesquieu nicht etwa die von Aristoteles als Königswissenschaft inthronisierte Staats- beziehungsweise Politikwissenschaft, ${ }^{51}$ sondern geht insbesondere mit der Politischen Theorie eine enge Verbindung dergestalt ein, dass die Politische Soziologie beschreibt, welche expansiven Ziele die Regimetypen haben, was wiederum mit deren in den Bereich der Politischen Theorie fallenden Prinzipien korreliert. So meint Montesquieu, Despotien strebten ob ihres Prinzips der Furcht nach Expansion, während die auf dem Prinzip der Ehre basierenden Monarchien zwar die Vergrößerung ihres Gebietes begehrten, nicht jedoch in grenzenlos expansiver Absicht. Montesquieus bevorzugte Regierungsform schließlich, die Republik, strebt, wenn sie eine solche bleiben will, nach Begrenzung ihres Gebietes, sei es aufgrund des demokratischen Prinzips der Tugend, sei es aufgrund des aristokratischen Prinzips der Mäßigung.

Hierin drückt sich Montesquieus irenisches Grundmotiv aus, den unaufhaltsamen gesellschaftlichen Modernisierungsprozess ohne bestandsgefährdende Friktionen gestalten zu wollen, indem nach den soziologischen Realisierbarkeitsbedingungen von Demokratisierungskonzeptionen gefragt wird. Die Betonung einer differenzierungstheoretischen Pointe von Montesquieus liberalem Staatsdenken korrigiert aber nicht nur eine staatsfeindliche, anti-politische Lesart soziologischer Differenzierungstheorie, sondern enthält aktuelle Einsichten in den Zusammenhang von Demokratie und Staatlichkeit.

\section{Von der Differenzierung der Demokratisierung zur Demokratisierung der Differenzierung}

Montesquieu konzipiert eine Differenzierung der Demokratisierung dergestalt, dass revolutionären Umwälzungen gewehrt und jede Gewalt, auch diejenige des Volkes über sich selbst, separiert und dadurch begrenzt wird. ${ }^{52}$ Dies beinhaltet ein Demokratieverständnis, in dem der Staat eine unverzichtbare, zentrale Rolle einnimmt ${ }^{53}$ während der Egalitarismus als paradoxes Prinzip eingeführt wird, das sich gerade dadurch erweisen und bewähren soll, dass es elitär überformt wird. So heißt es unter dem Titel »Worin die Liebe zum Staat in der Demokratie besteht« im 3. Kapitel des V. Buches Vom Geist der Gesetze:

»Die Liebe zum Staat in einer Demokratie ist die Liebe zur Demokratie, und die Liebe zur Demokratie ist die Liebe zur Gleichheit. Die Liebe zur Demokratie ist

51 Vgl. Aristoteles, Nikomachische Ethik. Übers. v. Franz Dirlmeier, Stuttgart 1983, S. $5 f$.

52 Aron, Hauptströmungen des soziologischen Denkens, aaO. (FN 6), S. 38.

53 Holmes, Differenzierung und Arbeitsteilung im Denken des Liberalismus, aaO. (FN 2), S. 19. 
weiter die Liebe zur Einfachheit. [...] Durch die Liebe zur Gleichheit wird in einer Demokratie der Ehrgeiz auf das eine Verlangen und eine Glück beschränkt, dem Vaterlande besser dienen zu können als die Mitbürger. Sie können ihm nicht alle gleiche Dienste leisten, aber alle sollen sie ihm gleichmäßig dienen [...]. So erwachsen die Unterschiede aus dem Gleichheitsgrundsatz dann, wenn er durch erfolgreichere Dienste und höhere Begabungen offenbar aufgehoben scheint. $\aleph^{54}$

Die scheinbar strikt egalitäre und auf sozio-moralische Qualitäten ausgerichtete Konzeption Montesquieus erweist sich also bei genauerem Hinsehen als Plädoyer für die Integration meritokratischer beziehungsweise aristokratischer Elemente. Der demokratische Egalitarismus ruht auf Elitismus. Der Weg zum Allgemeinwohl führt demnach nicht, wie bei Adam Smith, über die Freisetzung allgemeinen, »plebejischen« Gewinnstrebens zulasten staatlicher Intervention. Vielmehr sieht Montesquieu in dem aristokratischen Prinzip der Mäßigung gerade die Bedingung der Möglichkeit aktiven staatlichen Handelns. So hätten »die guten Demokratien«, Athen und Rom, »indem sie häusliche Einfachheit verlangen, für Staatsausgaben weit die Tore geöffnet ${ }^{55}$ Folgerichtig betont Montesquieu, was sich wie eine vorweggenommene Kritik des von George W. Bush propagierten »compassionate conservatism « ${ }^{56}$ liest:

»Ein paar Almosen, die man einem Bettler auf der Straße reicht, erfüllen die Verpflichtungen des Staates nicht, der allen Bürgern ein sicheres Auskommen, Nahrung, angemessene Kleidung und eine Lebensmöglichkeit schuldet, die der Gesundheit entspricht. « ${ }^{57}$

Montesquieu vertritt damit eine liberal-demokratische Auffassung, die auf aristokratischer Mäßigung ruht, und dies bestimmt auch die Logik der Gewaltenverteilung und damit des demokratischen Konstitutionalismus: Politische Machtverhältnisse sollen durch ein maßvolles Kräftegleichgewicht optimiert werden. Dies bedeutet, wie bereits an der Abgrenzung gegenüber Aristoteles gezeigt, dass der »Überbau « nur unter Rückgriff auf die sozialstrukturelle Basis konstruierbar ist. Und diesbezüglich sieht Montesquieu die Notwendigkeit einer »heterogenen und hierarchisch gegliederten Gesellschaft «. ${ }^{58}$ Denn $»$ in der Demokratie ist das Volk in gewisser Hinsicht der Monarch, in anderer Hinsicht Untertan. «9 $^{59}$ Dieses Demokratieverständnis Montesquieus lässt sich in moderner Terminologie folgendermaßen reformulieren: Demokratie bedeutet die Anerkennung einer staatlichen Herrschaftsordnung - und da jegliches Herrschaftsverhältnis auf Subordination beruht, mithin einer Hierarchie -, unter der Bedingung, dass sie auf Zeit gestellt und das Regierungshandeln zurechenbar ist, so dass Wahlen Sanktionsmöglichkeiten garantieren.

54 Montesquieu, Vom Geist der Gesetze, aaO. (FN 9), Bd. I, S. $63 f$.

55 Montesquieu, Vom Geist der Gesetze, aaO. (FN 9), Bd. I, S. 64.

56 Vgl. hierzu Karsten Fischer, Moralkommunikation der Macht. Politische Konstruktion sozialer Kohäsion im Woblfahrtsstaat, Wiesbaden 2006, S. $189 \mathrm{ff}$.

57 Montesquieu, Vom Geist der Gesetze, aaO. (FN 9), Bd. II, S. 158.

58 Aron, Hauptströmungen des soziologischen Denkens, aaO. (FN 6), S. 57.

59 Montesquieu, Vom Geist der Gesetze, aaO. (FN 9), Bd. I, S. 19. 
Mit Neoliberalismus hat Montesquieu mithin ebenso wenig im Sinn wie mit radikaldemokratischem Egalitarismus. Eher in Richtung der sogenannten »realistischen « Demokratietheorien weisend, intendiert Montesquieu, »who passes as a conservative breed of liberal $\ll^{60}$, nicht nur die angesprochene, bewahrende Differenzierung der Demokratisierung, sondern ebenso eine Demokratisierung der Differenzierung ${ }^{61}$ wie sie sich aus der Einsicht in das wechselseitige Bedingungsverhältnis zwischen konstitutioneller Demokratie und sozialer Komplexität infolge funktionaler Differenzierung ergibt.

Demokratie bedeutet gemäß diesem Verständnis Montesquieus die Legitimation von Entscheidungen durch ein Verfabren, das unter der Voraussetzung der Gleichbeit und der Transparenz die Zurechenbarkeit und also Sanktionierbarkeit dieser Entscheidungen garantiert. Politische Herrschaft ist demzufolge legitim, wenn die Machtunterworfenen die politischen Entscheidungen, deren Konsequenzen sie betreffen, den Machthabern ursächlich zurechnen können und aufgrund der Reversibilität der Machtverhältnisse ändern können. Auch in dieser demokratietheoretischen Pointe von Montesquieus Staatstheorie zeigt sich folglich die zentrale Bedeutung seiner Differenzierungsideen, die sich gleichermaßen auf die Ebene der funktionalen Primärdifferenzierung der Gesellschaft, die Verteilung der politischen Gewalten und die Leistungsmerkmale der Demokratie richten und sich auch auf das Verhältnis der politikwissenschaftlichen Teildisziplinen erstrecken.

\section{Zusammenfassung}

Montesquieus Schritt von der normativen politischen Theorie hin zur Politischen Soziologie beinhaltet Elemente einer Theorie sozialer Differenzierung, die auch seine Gewaltenverteilungslehre prägen. Verbunden mit seinem liberalen Öffentlichkeitskonzept, ermöglicht ihm dies eine modernisierungsskeptische Differenzierung der Demokratisierungsfrage, die das Verständnis und Verhältnis von Demokratie und Staatlichkeit ebenso betrifft wie das Verhältnis zwischen Regierungslehre, Politischer Soziologie und Politischer Theorie. In Äquidistanz zu den Antipoden Aristoteles und Hobbes wie auch zur Radikalität Rousseaus steht somit vor allem Montesquieus Denken für die Ermöglichung des liberalen Konstitutionalismus.

\section{Abstract}

Montesquieu's step from normative political theory towards political sociology includes elements of a theory of social differentiation, which also determine his idea of separation of powers. Together with his liberal concept of the public sphere, this

60 Holmes, Passions and Constraint. On the Theory of Liberal Democracy, aaO. (FN 22), S. 32 .

61 Vgl. Hans Joas, »Die Demokratisierung der Differenzierungsfrage. Die Krise des Fortschrittsglaubens und die Kreativität des kollektiven Handelns « in: Soziale Welt 41, 1990, S. 8-27. 
theory provides Montesquieu's differentiation of the democratization problem, resulting from his scepticism towards modernization. This is relevant for the understanding of and the relation both between democracy and statehood and between comparative government, political sociology and political theory. Thus, it is Montesquieu's political idea, equally distant from the antipodeans Aristotle and Hobbes as well as from Rousseau's radicalism, which facilitates liberal constitutionalism.

Karsten Fischer, Democracy and Differentiation in Montesquieu 